\title{
OPERATIONAL CALCULUS AND DIFFERENTIAL EQUATIONS WITH INFINITELY SMOOTH COEFFICIENTS
}

\author{
DENNIS NEMZER \\ Department of Mathematics \\ California State University, Stanislaus \\ 801 West Monte Vista Avenue \\ Turlock, CA 95380, U.S.A.
}

(Recelved May 2, 1989)

\begin{abstract}
A subring $M_{F}$ of the field of Mikusifisi operators is constructed as a countable union space. Some topological properties of $M_{F}$ are investigated. Then, the product of an infinitely differentiable function and an element of $M_{F}$ is given and is used to investigate operational equations with infinitely smooth coefficients.
\end{abstract}

KEY WORDS AND PHRASES. Mikusinski operator, infinitely differentiable, operational equation.

1980 AMS SUBJECT CLASSIFICATION CODE. Primary 44A40, Secondary 46F99, 34 A30.

1. INTRODUCTION.

The theory of generalized functions has been used successfully in solving problems in classical analysis as well as in simplifying the theory of differential equations (Mikusinski [1], Zemanian [2,3]). In the field of Mikusinski operators, the inability to define a sultable product of a function and an operator, see Stankovic [4], has had a limiting effect. For example, the theory and applications of ordinary differential equations have been restricted to differential equations having constant coefficients. In this note we will confine ourselves to the field $M$ of Mikusinski operators [1]. We will study a subring $M_{F}$ of Mikusinski operators in which the product of an infinitely smooth function and an operator in $M_{F}$ can be suitably defined. In Section 2 the construction of $M_{F}$ and a convergence in $M_{F}$ are studied. Then in Section 3, it is shown that an operational equation with infinitely smooth coefficients has only the classical solution. Some examples are then given to show that this is not the case if singularities are introduced.

2. THE CONSTRUCTION OF M F AND CONVERGENCE.

The ring of continuous complex-valued functions on $[0, \infty)$, denoted by $c$, with

addition and convolution $\left((f * g)(t)=\int_{0}^{t} f(t-u) g(u) d u\right)$ has no zero divisors. The quotient field of $C$ is denoted by $M$ and is called the field of Mikusinski operators. A typical element $x$ of $M$, called an operator, is written as $x=f / g$, where $f, g \in C$. 
The integral operator $\ell$ is the function defined as 1 for $t>0$ and 0 for $t<0$. The integral operator has the property that, for all $f \varepsilon C,\left(l^{\star} f\right)(t)=\int_{0}^{t} f(u) d u$. The inverse of $\ell$, denoted by $s$, is called the differentiation operator.

$\ell^{k}$ will denote $l * \ell * \ell * \ldots * * l$ (k terms), while $\phi^{(k)}$ will denote the $k^{\text {th }}$ derivative of $\phi$.

For $k=0,1,2, \ldots$, let $M^{k}=\left\{x \in M: e^{k}{ }_{x} \varepsilon C\right\}$. By endowing $M^{K}$ with the topology induced by the countable family of seminorms $\gamma_{k, m}(x)=\sup \left\{\left|\left(l^{k} \star_{x}\right)(t)\right|: 0<t<m\right\}$ for $m=1,2, \ldots M^{k}$ is a Fréchet space. Clearly, $x_{n}+x$ in $M^{k}$ if and only if for each $m>0, \ell^{k} \star_{n}+\ell^{k} \star_{x}$ uniformly on $[0, m]$.

Let $M_{F}$ be the countable union space $U M^{k}$. That is, $x$ is an element of $M_{F}$ if $x$ is an element of $M^{k}$ for some $k$. Also, a sequence $\left\{x_{n}\right\}$ in $M_{F}$ is said to converge to an element $x$ in $M_{F}$ if for some some $k, x_{n}, x \in M^{k} n=1,2, \ldots$ and $\left\{x_{n}\right\}$ converges to $x$ in the topology of $M^{k}$. Since each $M^{k}$ is complete, $M_{F}$ is sequentially complete. For a more detalled discussion of countable union spaces see [2].

Even though $M$ is considerably larger than $M_{F}$, $M_{F}$ contains many of the important operators needed for applications. For, by identifying the locally integrable function $f$ with the operator $\ell * f / \ell$, the collection of locally integrable functions can be identified with a subring of $M_{F}$. Moreover, $M_{F}$ contains all rational expressions in s.

We state without proof two lemmas.

LEMMA 2.1. Let $\left\{\phi_{n}\right\}$ be a sequence of positive functions such that:

(1) $\int_{-\infty}^{\infty} \phi_{n}(t) d t=1$ for all $n$ and (ii) supp $\phi_{n} \subseteq\left[0, \varepsilon_{n}\right]$, where $\varepsilon_{n}+0$

(supp $\phi_{n}$ is the closure of the set on which $\phi_{n}$ is not zero). Then for $f \in C$, and for each $m>0$, the sequence $\left\{\phi_{n} \star_{f}\right\}$ converges uniformly to $f$ on $[0, m]$.

LEMMA 2.2. Let $\left\{\phi_{n}\right\}$ be a sequence of functions such that on each interval $[0, m]$ $\phi_{n}+\phi$ uniformly. Then, for $f \varepsilon C$ and each interval $[0, m], \phi_{n} \star_{f}+\phi^{*} f$ uniformly.

$A$ subset $S$ of a countable union space $X$ is said to be dense if for each $x \in X$ there is some sequence $\left\{x_{n}\right\}$ in $S$ that converges to $x$.

THEOREM 2.3. $C$ is dense in $M_{F}$.

PROOF. Let $x \in M^{k}$ for some $k$. Let $\left\{\phi_{n}\right\}$ be a sequence of $k$-times continuously differentiable positive functions satisfying the following three properties:

(1) $\int_{-\infty}^{\infty} \phi_{n}(t) d t=1$ for all $n$; (ii) supp $\phi_{n} \subseteq[0,1 / n]$, for $n=1,2, \ldots$,

(iii) For each $n, \phi_{n}{ }^{(j)}(0)=0, j=0,1,2, \ldots, k$. Then, by Lemma 2.1 , for any continuous function $f,\left\{\phi_{n} \star_{f}\right\}$ converges uniformly to $f$ on compact subsets of $[0, \infty)$. Thus $\left[\left(e^{k} \star_{x}\right) \star_{\phi_{n}}(k)\right] * l^{k}, e^{k} \star_{x \in C}$ for all $n$ and $\left[\left(e^{k} \star_{x}\right) \star_{\phi_{n}}(k)\right] e^{k}=\left(e^{k} \star_{x}\right) \star_{n}+e^{k} \star_{x}$ 
uniformly on compact subsets of $[0, \infty)$. That is $\left\{l^{k} \star_{x} \star_{n}(k)\right\}$ converges to $x$ in $M_{F}$. Thus, $C$ is dense in $M_{F}$.

THEOREM 2.4. The mapping $M_{F}+M_{F}$ given by $x+x^{*} y$, where $y \varepsilon M_{F}$, is sequentially continuous.

PROOF. Let $\left\{x_{n}\right\}$ be a sequence in $M_{F}$ that converges to $x$. Thus, for some $k$, $\ell^{k} \star_{x_{n}}, l^{k} \star_{x} \in C$ for $n=1,2, \ldots$, and on each interval $[0, m]$ the sequence $\left\{l^{k} \star_{x_{n}}\right\}$ converges uniformly to $l^{k} \star_{x}$. Let $y \varepsilon M_{F}$. Then, for some $1, l^{1} \star_{y} \varepsilon C$. Hence, $e^{k+1} \star_{x_{n}}, l^{k+1} \star_{x} \in C$ for $n=1,2, \ldots$ and, by Lemma 2.2 , the sequence $\left\{l^{k+1} \star_{x_{n}}{ }_{y}\right\}$ converges uniformly on each interval $[0, m]$ to $e^{k+1} \star_{x} \star_{y}$. That $i s,\left\{x_{n}{ }^{\prime}\right\}$ converges to $x^{*} y$ in ${ }_{F}$. This establishes the theorem.

The collection of infinitely smooth complex-valued functions on $(-\infty, \infty)$ will be denoted by $\mathrm{C}^{\infty}$. We now define the product of an infinitely smooth function and an operator in $\mathrm{M}_{\mathrm{F}}$.

DEFINITION 2.5. For $\phi \varepsilon C^{\infty}$ and $x \in M^{k}$, the product of $\phi$ and $x$, denoted by $\phi \cdot x$, is given by

$$
\phi \cdot x=\sum_{j=0}^{k}(-1)^{j}\left(\begin{array}{l}
k \\
j
\end{array}\right) \frac{\phi^{(j)}\left(e^{k} x\right)}{e^{k-j}} \quad\left(\text { where } f / l^{0}=f\right)
$$

REMARKS 2.6. (i) If $\phi \varepsilon C^{\infty}$ and $f \varepsilon C$, then $\phi . f$ is ordinary multiplication. (ii) $1 \cdot x=x$ for $x \in M_{F}$.

It is not clear that multiplication is a well-defined operation. To see that it is, notice that if $n>m, f / l^{n}=g / l^{m}$ if and only if $f=\ell^{n-m} \star g$. Now, let

$$
F=\sum_{j=0}^{n+k}(-1)^{j}\left(\begin{array}{c}
n+k \\
j
\end{array}\right) e^{j} *\left[\phi^{(j)}\left(e^{k} \star_{f}\right)\right]-\sum_{j=0}^{n}(-1)^{j}\left(\begin{array}{l}
n \\
j
\end{array}\right) e^{k+j} *\left[\phi^{(j)} f\right],
$$

where $k$ is a nonnegative integer. After differentiating $F n+k$ times we obtain $F^{(n+k)}(t)=0$ for $t>0$ and $F^{(j)}\left(0^{+}\right)=0$ for $j=0,1,2, \ldots, n+k-1$. Thus, $F(t)=0$ for $t>0$. Therefore, $\phi \cdot\left(f / l^{n}\right)=\phi \cdot\left(e^{k} * f / l^{n+k}\right)$.

THEOREM 2.7. For $\phi, \psi \varepsilon C^{\infty}$ and $x \in M_{F},(\phi \cdot \psi) \cdot x=\phi \cdot(\psi \cdot x)$.

PROOF. Suppose that $\phi, \psi \varepsilon C^{\infty}$ and $x \varepsilon M^{k}$. Then,

$$
\begin{aligned}
& \phi \cdot(\psi \cdot x)=\sum_{n=0}^{k}(-1)^{n}\left(\begin{array}{l}
k \\
n
\end{array}\right) \sum_{j=0}^{k-n}(-1)^{j}\left(\begin{array}{c}
k-n \\
j
\end{array}\right) \frac{\phi^{(j)}\left[\psi^{(n)}\left(e^{k} \star x\right)\right]}{e^{k-n-j}} \\
& =\sum_{n=0}^{k} \sum_{j=0}^{n}(-1)^{n}\left(\begin{array}{l}
k \\
j
\end{array}\right)\left(\begin{array}{l}
k-j \\
n-j
\end{array}\right) \frac{\phi^{(n-j)} \psi^{(j)}\left(e^{k} * x\right)}{e^{k-n}}
\end{aligned}
$$

The last equality follows by the transformation $u=j+n$ and $v=n$.

Now,

$$
(\phi \cdot \psi) \cdot x=\sum_{n=0}^{k}(-1)^{n}\left(\begin{array}{l}
k \\
n
\end{array}\right) \frac{(\phi \psi)^{(n)}\left(e^{k} \star x\right)}{e^{k-n}} .
$$


By Leibniz's formula, we see that (2.1) and (2.2) are equivalent. Hence, the theorem is proved.

EXAMPLES 2.8 .

$$
\begin{aligned}
& \phi \cdot s^{n}=\sum_{j=0}^{n}(-1)^{j}\left(\begin{array}{c}
n \\
j
\end{array}\right) \phi^{(j)}(0) s^{n-j}, \quad \text { for } n=0,1,2, \ldots \\
& \text { (where } s^{0}=\delta, \delta=\ell / \ell \text { is the identity operator). }
\end{aligned}
$$

$$
t^{m} \cdot s^{n}= \begin{cases}0 & n<m \\ (-1)^{m} \frac{n !}{(n-m) !} s^{n-m} & n>m\end{cases}
$$

REMARKS. 2.9. (1) Example (i) follows by substituting the formula

$$
s^{n} \star_{\phi}=\phi^{(n)}+\sum_{k=0}^{n-1} \phi^{(k)}(0) s^{n-(k+1)} \quad \text { (see [1]) }
$$

into the definition for $\phi \cdot s^{n}$.

(1i) Example (ii) follows by substituting $\phi(t)=t^{m}$ into Example (i).

THEOREM 2.10. The mapping $M_{F}$ into $M_{F}$ given by $x \rightarrow \phi \cdot x$, where $\phi \varepsilon C^{\infty}$, is sequentially continuous.

PROOF. Suppose that $\left\{x_{n}\right\}$ converges to $x$ in $M_{F} \cdot$ That 18 , for some $k, \ell^{k} \star_{x_{n}}, \ell^{k} \star_{x} \in C n=1,2, \ldots$ and on each interval $[0, m],\left\{l^{k_{x_{n}}}\right\}$ converges uniformly to $\ell^{k} \star_{x}$.

Now, $\ell^{k} \star\left(\phi \cdot x_{n}\right)=\sum_{j=0}^{k}(-1)^{j}\left(\begin{array}{l}k \\ j\end{array}\right) \ell^{j} \star\left[\phi(j)\left(e^{k} \star_{x_{n}}\right)\right] \varepsilon c \quad$ for $n=1,2, \ldots$.

Moreover, for each $0<j<k$ and on each interval $[0, m],\left\{e^{j}\left(\phi^{(j)}\left(l^{k} \star_{x_{n}}\right)\right)\right\}$ converges uniformly to $\ell^{j} *\left(\phi^{(j)}\left(e^{k} \star_{x}\right)\right)$. Hence, $\left\{\phi \cdot x_{n}\right\}$ converges to $\phi \cdot x$ in $M_{F}$. This establishes the theorem.

If we consider $C^{\infty}$ as a subspace of $M_{F}$, then the mapping from $C^{\infty}$ into $M_{F}$ given by $\phi+\phi \cdot x$ is not sequentially continuous. To see this, let $\psi$ be any infinitely smooth positive function such that: (1) $\psi(0)=1$, (i1) $\psi(t)=0$ for $|t|>1$, and (iii) $\int_{-\infty}^{\infty} \psi(t) d t=1$. Now, for $n=1,2, \ldots$, let $\phi_{n}(t)=\psi(n t)$. Then, for each $n$ and for $t>0,0<\left(l^{*} \phi_{n}\right)(t)=\int_{0}^{t} \phi_{n}(u) d u=\frac{1}{n} \int_{0}^{n t} \psi(u) d u<1 / n$.

Thus, $\left\{\phi_{n}\right\}$ converges to 0 in $M_{F} \cdot$ But, for each $n, \phi_{n} \cdot \delta=\phi_{n}(0) \delta=\delta$. Hence, $\left\{\phi_{n} \cdot \delta\right\}$ does not converge to 0 in $M_{F}$.

In the theory of distributions, the countable family of seminorms $\gamma_{n, m}(\phi)=\sup \left\{\left|\phi^{(n)}(t)\right|:|t|<m\right\}, n=0,1,2, \ldots, m=1,2, \ldots$ is used to generate a topology for $C^{\infty}$. In the following theorem, we assume that $C^{\infty}$ is given this 
topology. The proofs of the next theorem and corollary are stralghtforward and thus omitted.

THEOREM 2.11. The mapping from $C^{\infty}$ into $M_{F}$ given by $\phi \rightarrow \phi \cdot x$ is sequentially continuous.

COROLLARY 2.12. Let $\phi(t)=\sum_{n=0}^{\infty} \alpha_{n} t^{n} \quad$ for $|t|<\infty$.

Then for $x \in M_{F}, \phi \cdot x=\sum_{n=0}^{\infty} \alpha_{n}\left(t^{n} \cdot x\right)$.

3. DIFFERENTIAL EQUATIONS AND OPERATIONAL EQUATIONS.

It is known that the only solutions, within the framework of distribution theory, to a linear homogeneous ordinary differential equation with infinitely smooth coefficients are the classical ones. But, when the coefficients have singularities, there may be other distributional solutions (see [5] Littlejohn and Kanwal, and Wiener [6]). In this section we will show that in $M_{F}$ the situation is similar. For simplicity, we will only consider second order differential and operational equations. However, what follows is also true for nth order equations.

When solving the inftial value problem $y^{\prime \prime}+\alpha_{1} y^{\prime}+\alpha_{2} y=0, y(0)=\beta_{1}$ and $y^{\prime}(0)=\beta_{2}$ using operational methods, the corresponding operational equation is $s^{2} y+\alpha_{1}\left(s^{*} y\right)+\alpha_{2} y=\left(\beta_{2}+\alpha_{1} \beta_{1}\right) \delta+\beta_{1} s$. This follows by the formula given in Remark 2.9(1). By a similar argument, the differential equation $y^{\prime \prime}+\phi_{1} y^{\prime}+\phi_{2} y=0$, where $\phi_{1}, \phi_{2} \in C^{\infty}$, corresponds to the operational equation

$$
s^{2} \star_{x}+\phi_{1} \cdot(s * x)+\phi_{2} \cdot x=\left(\beta_{2}+\beta_{1} \phi_{1}(0)\right) \delta+\beta_{1} s
$$

where $y(0)=B_{1}$ and $y^{\prime}(0)=B_{2} \cdot$ Thus, we will consider second order operational equations of the form $s^{2} \star_{x}+\phi_{1} \cdot\left(s^{*} x\right)+\phi_{2} \cdot x=\alpha_{1} \delta+\alpha_{2} s$, where $\alpha_{1}$ and $\alpha_{2}$ are constants and $\phi_{1}, \phi_{2} \in C^{\infty}$.

THEOREM 3.1. Let $\alpha_{1}$ and $\alpha_{2}$ be constants and $\phi_{1}, \phi_{2} \varepsilon C^{\infty}$. The only solution to the operational equation $s^{2} \star_{x}+\phi_{1} \cdot\left(s^{*} x\right)+\phi_{2} \cdot x=\alpha_{1} \delta+\alpha_{2} s$ is the classical solution to the corresponding differential equation.

PROOF. Let $x \in M^{k}$ such that

$$
\begin{aligned}
s^{2} \star_{x} & +\sum_{j=0}^{k+1}(-1)^{j}\left(\begin{array}{c}
k+1 \\
j
\end{array}\right) \frac{\phi_{1}^{(j)}\left(e^{k} \star_{x}\right)}{e^{k+1-j}}+\sum_{j=0}^{k}(-1)^{j}\left(\begin{array}{l}
k \\
j
\end{array}\right) \frac{\phi_{2}(j)}{\left.e^{k} e^{k}\right)} \\
& =\alpha_{1} \delta+\alpha_{2} s .
\end{aligned}
$$

By convolving both sides with $\ell^{k+2}$ we obtain

$$
\begin{aligned}
& e^{k} x_{x}+\sum_{j=0}^{k+1}(-1)^{j}\left(\begin{array}{c}
k+1 \\
j
\end{array}\right) e^{j+1} \star\left[\phi_{1}{ }^{(j)}\left(e^{k} \star_{x}\right)\right] \\
& +\sum_{j=0}^{k}(-1)^{j}\left(\begin{array}{c}
k \\
j
\end{array}\right) e^{j+2} \star\left[\phi_{2}^{(j)}\left(e^{k} \star_{x}\right)\right]=\alpha_{1} e^{k+2}+\alpha_{2} e^{k+1} .
\end{aligned}
$$


Now, notice that each term, except the first $\ell^{k} \star_{x}$, in (3.1) is differentiable on $(0, \infty)$. Hence, $\ell^{k}{ }_{x}$ is also differentiable on $(0, \infty)$. By an inductive argument, we obtain $\ell^{k} \star_{x} \varepsilon C^{\infty}(0, \infty)$.

After differentiating (3.1) $k+2$ times we obtain

Moreover, $\quad\left(e^{k} x_{x}\right)^{(k+2)}+\phi_{1}\left(l^{k} \star_{x}\right)^{(k+1)}+\phi_{2}\left(l^{k} \star_{x}\right)^{(k)}=0$.

Now let

$$
g=\left(l^{k} *_{x}\right)^{(k)}
$$

Then, $g$ is the unique solution to the initial value problem $g^{\prime \prime}+\phi_{1} g^{\prime}+\phi_{2} g=0$, $g(0)=\alpha_{2}$, and $g^{\prime}(0)=\alpha_{1}-\phi_{1}(0) \alpha_{2} \cdot$ By (3.2) and (3.3), $\ell^{k} \star_{x}=\ell^{k} \star_{g}$. That 18 , $x=g, g(0)=\alpha_{2}, g^{\prime}(0)=\alpha_{1}-\phi_{1}(0) \alpha_{2}$. This establishes the theorem.

The following examples demonstrate that the situation is different when singularities are allowed in the coefficients. By using the formula given in Example $2.8(11)$, it is easy to verify that $x$ is a solution to the corresponding operational equation.

EXAMPLES 3.2 .

(i) $x=\delta$ is a solution to $t \cdot\left(s^{2}{ }^{*} x\right)+(2-t) \cdot(s * x)-x=0$.

(i1) $x=\delta-s$ is a solution to $t \cdot\left(s^{2}{ }^{*} x\right)+(3-t) \cdot\left(s{ }^{*}\right)-x=0$.

REFERENCES

1. MIKUSINSKI, J., Operational Calculus, Pergamon Press, Oxford, 1959.

2. ZEMANIAN, A.H., Generalized Integral Transformations, Dover Publications, New York, 1987.

3. ZEMANIAN, A.H., Distribution Theory and Transform Analysis, Dover Publications, New York, 1987.

4. STANKOVIC, B., On the Impossibility of Extending the Product of Continuous Functions to the Field of Mikusinski Operators, Univ. Novi. Sad. 12 (1982), $1-6$.

5. LITTLEJOHN, L.L. and KANWAL, R.P., Distributional Solutions of the Hypergeometric Differential Equation, J. Math. Anal. Appl. 122 (1987), 325345.

6. WIENER, J., Generalized Function Solutions of Differential and Functional Differential Equations, J. Math. Anal. Appl. 88 (1982), 170-182. 


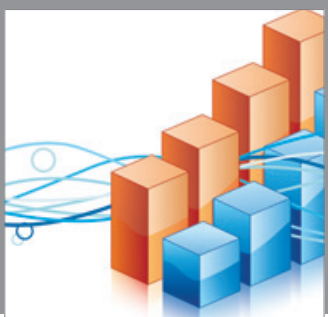

Advances in

Operations Research

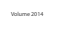

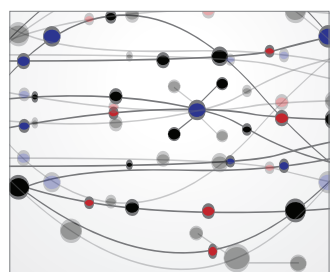

\section{The Scientific} World Journal
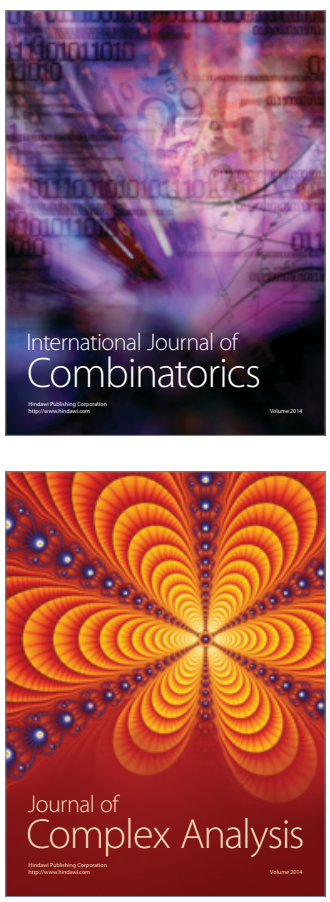

International Journal of

Mathematics and

Mathematical

Sciences
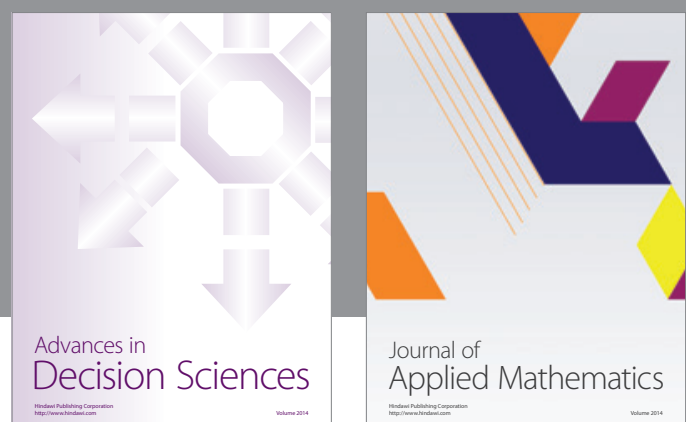

Journal of

Applied Mathematics
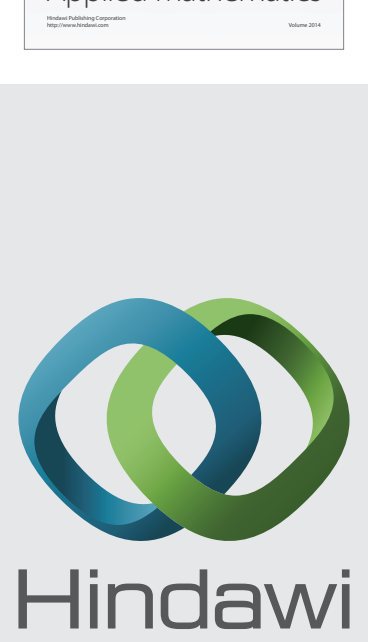

Submit your manuscripts at http://www.hindawi.com
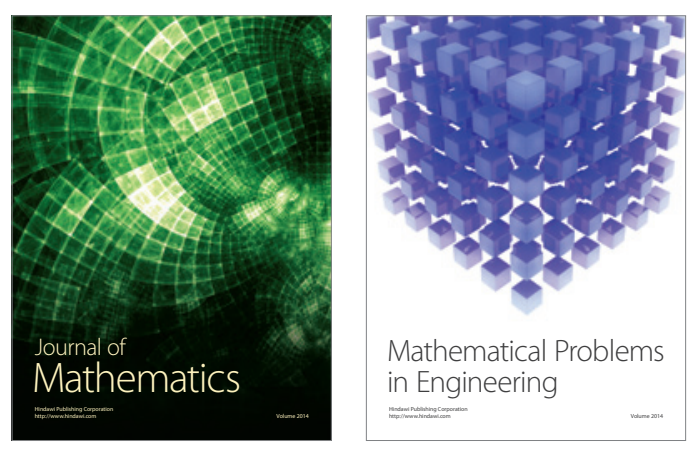

Mathematical Problems in Engineering
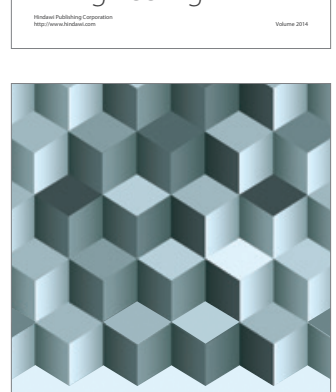

Journal of

Function Spaces
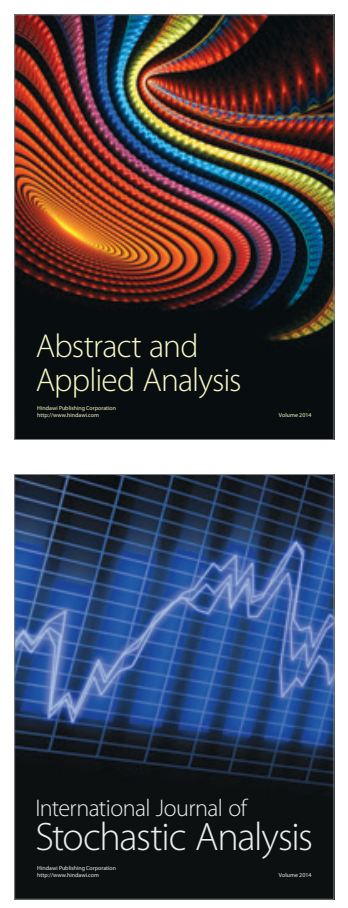

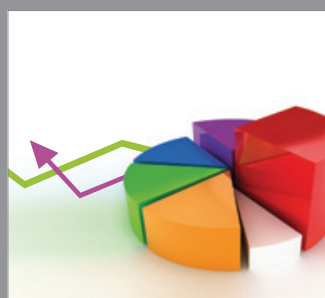

ournal of

Probability and Statistics

Promensencen
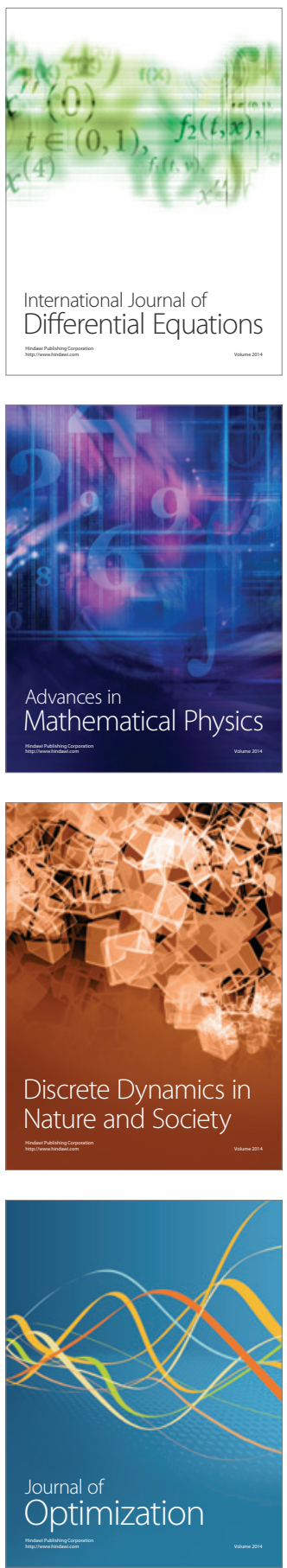